\title{
Control Design for One Class of Uncertain Metzler-Takagi-Sugeno Time-Delay Systems
}

\author{
Dušan KROKAVEC ${ }^{1}$ and Anna FILASOVÁ \\ Technical University of Košice, Slovakia
}

\begin{abstract}
This paper presents a new approach to synthesize the control for one class of uncertain Metzler-Takagi-Sugeno time-delay systems. The structural parametric constraints of the closed-loop system, their diagonal matrix representations as well as the interval system parameter bounds are accounted into an associated set of the linear matrix inequalities. After sorting out the relevant preliminaries in the uncertain structure of the Metzler systems and the specific properties of the timedelay positive system representations, the design conditions reflecting quadratic system stability of the considered system class are proven in the matrix inequality framework. The main result of the control law parameter design is shown in detail by the numerical example in order to characterize potential adaptation of the method for purely Metzler matrix parameter structures.
\end{abstract}

Keywords. Metzler-Takagi-Sugeno systems, positive systems, quadratic stability, point state delay, diagonal stabilization, linear matrix inequalities.

\section{Introduction}

Time-delay systems are used to model the time lag phenomenon in thermodynamics [1] and ecology [2] and in modeling of interactions between species in mathematical biology [3]. In order to control, a number of approaches are proposed to solve the problems fixed with time-delay systems (see, e.g., [4]). In this paper the time-delay phenomena is considered as a point delay in the state vector [5].

Due to uncertainty existence, the uncertain plant stabilization have attracted many attentions to solve simultaneously this problem, reflecting LQR control [6], $\mathrm{H}_{2}$ cost control [7] and $\mathrm{H}_{\infty}$ control principle [8]. In the sense of these prerequisites along with techniques for simultaneous quadratic stabilization of time-delay systems, the key concepts in stabilization and tracking conditions have been developed in [9] and the delay-dependent stabilization has been investigated in [10].

Positive systems represent the dynamical plants whose states and outputs have to be positive [11], [12]. The problem of positive systems usually deals with positive control structure and positive system state estimation [13]. Since existence of the system posi-

\footnotetext{
${ }^{1}$ Corresponding Author: Dušan Krokavec, Department of Cybernetics and Artificial Intelligence, Faculty of Electrical Engineering and Informatics, Technical University of Košice, Letná 9, 042 00, Košive Slovakia; E-mail: dusan.krokavec@tuke.sk
} 
tivity depends on the constraints in nonnegativity of the system matrix parameters, constrained design approaches are proposed to solve these problems [14]. Among the main features of positive systems can be mentioned the facts that the stability of delayed positive systems does not depend on the amplitude of delays [15], the asymptotical stability of positive systems is equivalent to diagonal stability (systems are not only stable but also positive) [16] and the system positivity can be interpreted through the model parametric constraints [17]. Since these models prioritize theory of matrices of Metzler structure [18], such systems are denoted oftentimes as Metzler systems. A similar approach one can found in development of control for the Metzler time-delayed systems [19].

Using the Takagi-Sugeno fuzzy model [20] a nonlinear system is represented as the collection of fuzzy rules, where each rule utilizes the local dynamics by a linear system model. Since Takagi-Sugeno fuzzy models can well approximate a large class of interval nonlinear systems the relevant design methodologies exploit fully advantage of the modern control theory, especially in the constrained control of positive T-S systems with delays [21] and in the positive system state estimation and fault diagnosis [22].

From the previous overview of methods and principles it can see hove many different aspects have to be covered in the control design for uncertain Metzler-Takagi-Sugeno time-delay systems. Although the principle of diagonal stabilization provides a way to represent the parametric constraints of linear positive systems using the linear programming method [23], its direct adaptation to this system class is hardly limited. To overcome these limitations an original, new approach is established to give explicit design conditions in the form of linear matrix inequalities (LMI) to guaranty the strictly positive closed-loop system matrices if the uncertain system model takes the time-delay strictly Metzler-Takagi-Sugeno form. Note that, this LMIs structure cover the system structural constraints, the parameter uncertainties, the point-delay in system state, the quadratic stability and the diagonal stabilisation principle. The primary goal is to find the controller for stabilisation of the system under consideration while being consistent with the positive configuration. Although such defined class of systems prescribes the set of strong structural parametric constraints, the proposed design conditions allow the numerical solution in straightforward access. It is authors' belief that further extensions can be done through the given theoretical framework.

The remaining of the article is lay out as follows: Section 2 deals with the problem formulation and representative preliminaries related to Metzler-Takagi-Sugeno timedelay dynamic systems. The control problem, the resulting system parameter constraint formats and the relevant synthesis formulation are given in Section 3. The performance of the proposed technique and the system parameters representation are illustrated by carrying out a detailed numerical example in Section 4, also supporting related outlines of new points of view and conclusions, drafted in Section 5.

For sake of convenience, throughout the paper used notations reflect usual conventionality so that $x^{\mathrm{T}}, X^{\mathrm{T}}$ denotes the transpose of the vector $x$, and the matrix $X$, respectively, $X^{-1}, \rho(X)$ signifies the inverse and the eigenvalue spectrum of a square matrix $X$, respectively, for a symmetric square matrix $X \prec 0$ means that $X$ is negative definite matrix, diag $[\cdot]$ marks the elements of a (block) diagonal matrix, $*$ represents the block in a square symmetric matrix that is readily inferred by the matrix symmetry, the symbol $I_{n}$ indicates the $n$-th order unit matrix, $\mathbb{R}\left(\mathbb{R}_{+}\right)$qualifies the set of (nonnegative) real numbers, $\mathbb{R}_{+}^{n \times r}$ refers to the set of $n \times r$ positive (nonnegative) real matrices and $\mathrm{M}_{-+}^{n \times n}$ is the set of (strictly or pure) Metzler matrices. 


\section{Basic Preliminaries}

The system class under consideration is multi-input, multi-output (MIMO) uncertain Metzler-Takagi-Sugeno time-delay dynamic systems, represented in state-space form as

$$
\begin{gathered}
\dot{q}(t)=\sum_{i=1}^{s} \mathrm{~h}_{i}(\vartheta(t))\left(A_{i}+\Delta_{i} A_{i}(t)\right) q(t)+\left(A_{s}+\Delta A_{s}(t)\right) q(t-\tau)+(B+\Delta B(t)) u(t), \\
y(t)=C q(t),
\end{gathered}
$$

where the point delay in the state vector is constant and satisfies the condition $0<\tau \in$ $\mathbb{R}_{+}$, the system input variable vector $u(t) \in \mathbb{R}^{r}$ and the uncertainties $\Delta A_{i}(t), \Delta B(t) \Delta A_{s}(t)$ are matching uncertainties. The system trajectory $q(t) \in \mathbb{R}_{+}^{n}$, and the output $y(t) \in \mathbb{R}_{+}^{m}$ are nonnegative, $C \in \mathbb{R}_{+}^{m \times n}, B, \Delta B(t) \in \mathbb{R}_{+}^{n \times r}$ and $\Delta A_{i}(t), \Delta A_{s}(t) \in \mathbb{R}_{+}^{n \times n}$ are nonnegative matrices, $A, A_{s} \in \mathbb{M}_{-+}^{n \times n}$ are strictly Metzler and $r=m$. Moreover, $\mathrm{h}_{i}(\theta(t))$ is averaging weight for the $i$-th rule, representing the normalized grade of membership, where

$$
0 \leq \mathrm{h}_{i}(\vartheta(t)) \leq 1, \quad \sum_{i=1}^{s} \mathrm{~h}_{i}(\vartheta(t))=1 \quad \text { for all } i \in\langle 1, s\rangle,
$$

while $s$ is the number fuzzy rules (linear sub-models) and

$$
\vartheta(t)=\left[\theta_{1}(t) \theta_{2}(t) \cdots \theta_{q}(t)\right]
$$

is $q$-dimensional vector of premise variables, where the premise variables are measurable. More details can be found, e.g., in [24], [25].

Definition 1. [18] A square matrix $A \in \mathbb{M}_{-+}^{n \times n}$ is purely Metzler if its diagonal elements are negative and its off-diagonal elements are nonnegative. A Metzler matrix $A \in \mathbb{M}_{-+}^{n \times n}$ is strictly Metzler if its diagonal elements are negative and its off-diagonal elements are positive. A Metzler matrix is stable if it is Hurwitz.

Definition 2. [26] Given any nonnegative initial condition $\phi(t) \in \mathbb{R}_{+}^{n}$ such that $q(t)=$ $\phi(t)$ for $-\tau \leq t \leq 0$, the externally unforced system (1) is said to be positive if the corresponding trajectory is nonnegative $\left(q(t) \in \mathbb{R}_{+}^{n}\right.$ for all $\left.t \geq 0\right)$.

Definition 3. (adapted from [8]) If the uncertainties of the system (1), (2) satisfy for every time-instant $t>0$ the condition

$$
\left.\left.\left.\left[\Delta A_{i}(t)\right) \Delta B(t)\right) \Delta A_{s}(t)\right)\right]=M \Xi(t)\left[N_{a i} N_{b} N_{s}\right],
$$

where $M \in \mathbb{R}_{+}^{n \times s}, N_{a i} \in \mathbb{R}_{+}^{p \times n}, N_{b} \in \mathbb{R}_{+}^{p \times r}, N_{s} \in \mathbb{R}_{+}^{p \times n}$ are known nonnegative real matrices characterizing the uncertainties structure, $\Xi(t) \in \mathbb{R}^{s \times p}$ is a time varying matrix satisfying the bound

$$
\Xi^{\mathrm{T}}(t) \Xi(t) \leq I_{p}
$$

and the elements of $\Xi(t)$ are Lebesgue measurable, then the uncertainties $\Delta A_{i}(t), \Delta B(t)$ $\Delta A_{s}(t)$, are matching uncertainties.

Note, as a rule, for point time-delay Metzler-Takagi-Sugeno systems with strictly positive $A_{i}, A_{s}$ and nonnegative $B$ all the matrices $M \in \mathbb{R}_{+}^{n \times s}, N_{a i} \in \mathbb{R}_{+}^{p \times n}, N_{b} \in \mathbb{R}_{+}^{p \times r}$, $N_{s} \in \mathbb{R}_{+}^{p \times n}$ have to be non-negative.

Since Metzler-Takagi-Sugeno systems are defined to satisfy affine combination of matrix elements [27], this suggests the following. 
Lemma 1. (adapted from [26]) Assuming that the externally unforced system (1) is asymptotically stable and the matrices $A_{s} \in \mathbb{M}_{-+}^{n \times n}$ and $A_{i} \in \mathbb{M}_{-+}^{n \times n}$ for $i=1, \ldots, s$ are strictly Metzler then the composed matrix

$$
A_{i}^{\diamond}=A_{i}+A_{s}
$$

is strictly Metzler and Hurwitz.

Remark 1. Obviously, if all matrices in (7) are strictly Metzler then their sum must have the form of a strictly Metzler matrix. In dependence on positions of zero elements in these matrices, there can be a strictly Metzler matrix $A_{i}^{\diamond}$ even for the case where both matrices are only purely Metzler, or one from them is strictly Metzler and the other is purely Metzler. This means different interpretations of the tasks of analysis and synthesis of such class of systems and leads to solutions with different parametric boundaries on Metzler structures [4], [28], [29]. Unless otherwise stated below, the both above given matrices in (7) are considered to be strictly Metzler for all $i$.

Remark 2. In general, a strictly Metzler matrix $A \in \mathbb{M}_{-+}^{n \times n}$ is so confronted with $n^{2}$ boundaries implying from the structural constraints

$$
a_{l h}<0, l=h, \quad a_{l h}>0, l \neq h, \quad \forall l, h \in\langle 1, n\rangle .
$$

This just means in consequence to apply diagonal stabilization principle [30] in control or observer design task. This principle can be adapted for the control design task if a strictly Metzler matrix $A \in \mathrm{M}_{-+}^{n \times n}$. is represented with the following rhombic form, constructed by circular shifts of the rows of the strictly Metzler A as follows

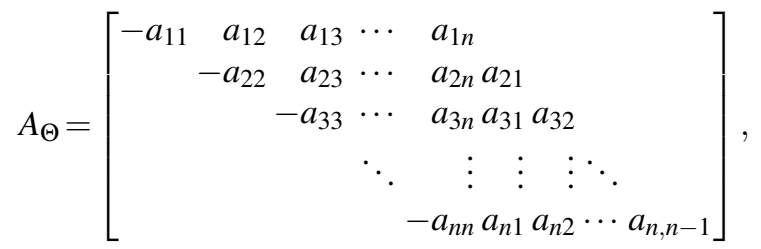

where the diagonal matrices

$$
\begin{gathered}
A_{\Theta}(l, l)=\operatorname{diag}\left[-a_{11}-a_{22} \cdots-a_{n n}\right] \prec 0, \\
A_{\Theta}(l, l+h)=\operatorname{diag}\left[a_{1,1+h} \cdots a_{n-h, n} a_{n-h+1,1} \cdots a_{n, h}\right] \succ 0, \quad h=1, \ldots, n-1,
\end{gathered}
$$

are related to diagonals of $A_{\Theta}$ and $\Theta=(1 \leftrightarrow n) / n$ notes summation in the sense of the sum of modulo $(n+1)$.

Moreover, it can see that generally $n^{2}$ parametric Metzler constraints (8) can be defined by the negativeness of (10) and positiveness of the set of diagonal matrices (11).

Definition 4. [31] A square matrix $L \in \mathbb{R}_{+}^{n \times n}$ is the permutation matrix if exactly one element in each column and each row is equal to 1 and all others are equal to 0 . The permutation matrix $L$ is called circulant if

$$
L=\left[\begin{array}{cc}
0^{\mathrm{T}} & 1 \\
I_{n-1} & 0
\end{array}\right] .
$$

Remark 3. If $X \in \mathbb{R}^{n \times n}$ is a diagonal matrix and $L$ is the circulant permutation matrix (12) then

$$
L^{\mathrm{T}} \operatorname{diag}\left[x_{1} x_{2} \cdots x_{n}\right] L=\operatorname{diag}\left[x_{2} \cdots x_{n} x_{1}\right]
$$


Lemma 2. [32] Let the matrix $A \in \mathbb{M}_{-+}^{n \times n}$ is strictly Metzler then it is Hurwitz if and only if there exists the positive definite diagonal matrix $S \in \mathbb{R}_{+}^{n \times n}$ that for $h=1, \ldots, n-1$ and the circulant $L \in \mathbb{R}_{+}^{n \times n}$ the following sets of LMIs is feasible

$$
\begin{aligned}
S \succ 0, & S A^{\mathrm{T}}+A S \prec 0, \\
A_{\Theta}(l, l) S \prec 0, & L^{h} A_{\Theta}(l, l+h) L^{h \mathrm{~T}} S \succ 0 .
\end{aligned}
$$

Lemma 3. [33] If the strictly Metzler $A \in \mathbb{R}_{-+}^{n \times n}$, the nonnegative $B \in \mathbb{R}_{+}^{n \times r}$ and a positive $K \in \mathbb{R}_{+}^{r \times n}$ guarantee that $A_{c}=A-B K \in \mathbb{R}_{-+}^{n \times n}$ is strictly Metzler then it is satisfied for the defined $h$ and $j$

$$
A_{c}=\sum_{h=0}^{n-1}\left(A_{\Theta}(l, l+h)-\sum_{j=0}^{r} B_{d j} K_{d j h}\right) L^{h \mathrm{~T}},
$$

where with $K_{d j}, B_{d j} \in \mathbb{R}_{+}^{n \times n}$

$$
\begin{gathered}
A_{\Theta}(l, l)-\sum_{j=0}^{r} B_{d j} K_{d j} \prec 0, \\
A_{\Theta}(l, l+h)-\sum_{j=0}^{r} B_{d j} K_{d j h} \succ 0, \\
K=\left[\begin{array}{c}
k_{1}^{\mathrm{T}} \\
\vdots \\
k_{r}^{\mathrm{T}}
\end{array}\right], \quad K_{d j}=\operatorname{diag}\left[k_{j}^{\mathrm{T}}\right]=\operatorname{diag}\left[k_{j 1} \cdots k_{j n}\right], \quad K_{d j h}=L^{h \mathrm{~T}} K_{d j} L^{h} \\
B=\left[\begin{array}{lll}
b_{1} & \cdots & \left.b_{r}\right], \quad B_{d j}=\operatorname{diag}\left[b_{j}\right]=\operatorname{diag}\left[b_{j 1} \cdots b_{j n}\right]
\end{array}\right.
\end{gathered}
$$

Note, (17), (18) force parameter constraints of the Metzler $A_{c}$ and do not guaranty that $A_{c}$ is Hurwitz. Equation (16) together with (19)-(20) give the relations between the square and the rhombic matrix representation of $A_{c}$.

Summarizing, in this section are fixed the basis notations and terminologies used in the paper, clarified the necessary background theoretical preliminaries from the assorted points of interpretation and recalled a number of relevant definitions. In order to commonly formalize control synthesis for the uncertain Metzler-Takagi-Sugeno time-delay systems in the following section, everything is defined on the common state-space basis.

\section{Control Design for the Class of Uncertain Metzler-Takagi-Sugeno Time-delay Systems}

This section presents the conditions on control existence for considered class of systems with constant time-delays and the LMI based formulation for computing the control law parameters. The considered problem is connected with the control law

$$
u(t)=-K q(t)-K_{s} q(t-\tau)
$$

such that in the closed-loop structure 


$$
\begin{aligned}
\dot{q}(t) & =\sum_{i=1}^{s} \mathrm{~h}_{i}(\vartheta(t))\left(A_{i}-B K\right) q(t)+\sum_{i=1}^{s} \mathrm{~h}_{i}(\vartheta(t))\left(\Delta A_{i}(t)-\Delta B(t) K\right) q(t)+ \\
& +\left(A_{s}-B K_{s}\right) q(t-\tau)+\left(\Delta A_{s}(t)-\Delta B(t) K_{s}\right) q(t-\tau) \\
& =\sum_{i=1}^{s} \mathrm{~h}_{i}(\vartheta(t)) A_{c i} q(t)+\sum_{i=1}^{s} \mathrm{~h}_{i}(\vartheta(t)) A_{\Delta c i}(t) q(t)+A_{c s} q(t-\tau)+A_{\Delta c s}(t) q(t-\tau),
\end{aligned}
$$

where

$A_{c i}=A_{i}-B K, A_{\Delta c i}(t)=\Delta A_{i}(t)-\Delta B(t) K, A_{c s}=A_{s}-B K_{s}, \Delta A_{c s}(t)=\Delta A_{s}(t)-\Delta B(t) K_{s}$,

the matrices $A_{c i}, A_{c s}, A_{c i}^{\circ}, A_{c s}^{\circ} \in \mathbb{R}_{-+}^{r \times n}$ are strictly Metzler, the control law gains $K, K_{s} \in$ $\mathbb{R}_{+}^{r \times n}$ are positive and

$$
A_{c i}^{\circ}=A_{c i}+A_{c s}
$$

are strictly Metzler and Hurwitz for all $i$.

The relation (5) then admits the uncertainty models if $p=r$

$$
\begin{aligned}
& \Delta A_{c i}(t)=M \Xi(t)\left(N_{a i}-N_{b} K\right)=M \Xi(t) N_{c i}, \\
& \Delta A_{c s}(t)=M \Xi(t)\left(N_{s}-N_{b} K_{s}\right)=M \Xi(t) N_{c s} .
\end{aligned}
$$

where

$$
N_{c i}=N_{a i}-N_{b} K, \quad N_{c s}=N_{s}-N_{b} K_{s} .
$$

Reflecting the diagonal stabilisation principle, then to the above introduced relations (19), (20) the additional terms are defined

$$
\begin{aligned}
& A_{c i}=A_{i}-\sum_{j=1}^{r} b_{j} k_{j}^{\mathrm{T}}=A_{i}-\sum_{j=1}^{r} B_{d j} l_{n} l_{n}^{\mathrm{T}} K_{d j} \\
& A_{c s}=A_{s}-\sum_{j=1}^{r} b_{j} k_{s j}^{\mathrm{T}}=A_{s}-\sum_{j=1}^{r} B_{d j} l_{n} l_{n}^{\mathrm{T}} K_{s d j} \\
& N_{c i}=N_{a i}-\sum_{j=1}^{r} n_{b j} k_{j}^{\mathrm{T}}=N_{a i}-\sum_{j=1}^{r} N_{b d j} l_{r} l_{n}^{\mathrm{T}} K_{d j} \\
& N_{c s}=N_{s}-\sum_{j=1}^{r} n_{b j} k_{s j}^{\mathrm{T}}=N_{s}-\sum_{j=1}^{r} N_{b d j} l_{r} l_{n}^{\mathrm{T}} K_{s d j} \\
& A_{\Theta i}(l, l)=\operatorname{diag}\left[\begin{array}{llll}
-a_{i 11} & -a_{i 22} & \cdots & -a_{i n n}
\end{array}\right], \\
& A_{\Theta i}(l, l+h)=\operatorname{diag}\left[\begin{array}{llllll}
a_{i, 1,1+h} & \cdots & a_{i, n-h, n} & a_{i, n-h+1,1} & \cdots & a_{i, n h}
\end{array}\right], \\
& A_{\Theta s}(l, l)=\operatorname{diag}\left[\begin{array}{llll}
-a_{s 11} & -a_{s 22} & \cdots & -a_{s n n}
\end{array}\right],
\end{aligned}
$$

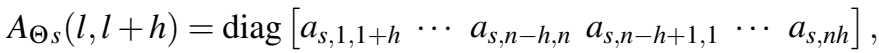

$$
\begin{aligned}
& K_{s}=\left[\begin{array}{c}
k_{s 1}^{\mathrm{T}} \\
\vdots \\
k_{s r}^{\mathrm{T}}
\end{array}\right], \quad K_{s d j}=\operatorname{diag}\left[k_{s j}^{\mathrm{T}}\right]=\operatorname{diag}\left[k_{s j 1} \cdots k_{s j n}\right], \quad K_{s d j h}=L^{h \mathrm{~T}} K_{s d j} L^{h} \\
& N_{b}=\left[\begin{array}{lll}
n_{b 1} & \cdots & n_{b p}
\end{array}\right], \quad N_{b d j}=\operatorname{diag}\left[n_{b j}\right]=\operatorname{diag}\left[n_{b j 1} \cdots n_{b j p}\right] . \\
& l_{n}^{\mathrm{T}}=\left[\begin{array}{llll}
1 & 1 & \cdots & 1
\end{array}\right], \quad l_{r}^{\mathrm{T}}=\left[\begin{array}{llll}
1 & 1 & \cdots & 1
\end{array}\right], \quad l_{n} \in \mathbb{R}_{+}^{n} \cdot l_{r} \in \mathbb{R}_{+}^{r}
\end{aligned}
$$

and (7) is reformulated as:

$$
A_{c i}^{\circ}=A_{c i}+A_{c s}
$$


To simply constructing under defined matching uncertainties the following lemma can be applied.

Lemma 4. [34] Let the real matrices $X, Y, Z$ of consistent dimensions are bound by the relation

$$
Z=X^{\mathrm{T}} Y+Y^{\mathrm{T}} X
$$

then for positive $\gamma \in \mathbb{R}_{+}$the following inequality is satisfied

$$
Z \preceq \gamma^{-1} X^{\mathrm{T}} X+\gamma Y^{\mathrm{T}} Y .
$$

Thus, considering (19), (20), (32)-(39) and the circulant $L \in \mathbb{R}_{+}^{n \times n}$ from (12) it can prove the following.

Theorem 1. If for strictly Metzler $A_{i}, A_{s} \in \mathbb{M}_{-+}^{n \times n}$ and nonnegative $B \in \mathbb{R}_{+}^{n \times r}, M \in \mathbb{R}_{+}^{n \times s}$, $N_{a i} \in \mathbb{R}_{+}^{p \times n}, N_{b} \in \mathbb{R}_{+}^{p \times r}, N_{s} \in \mathbb{R}_{+}^{p \times n}$ there exist positive definite diagonal matrices $S, R_{j}, R_{s j} \in \mathbb{R}_{+}^{n \times n}$ and a positive scalar $\delta \in \mathbb{R}_{+}$such that for $h=1, \ldots, n-1, i=1, \ldots, s$ is feasible the following set of linear matrix inequalities

$$
\begin{aligned}
& S \succ 0, \quad R_{j} \succ 0, \quad R_{s j} \succ 0, \quad \delta>0, \\
& {\left[\begin{array}{cc}
{\left[\left(A_{i}+A_{s}\right) S-\sum_{j=1}^{r} B_{d j} l_{n} l_{n}^{\mathrm{T}}\left(R_{j}+R_{s j}\right)\right]+[*]+\delta M M^{\mathrm{T}}} & * \\
\left(N_{a i}+N_{s}\right) S-\sum_{j=1}^{r} N_{b d j} l_{r} l_{n}^{\mathrm{T}}\left(R_{j}+R_{s j}\right) & -\delta I_{r}
\end{array}\right] \prec 0,} \\
& A_{\Theta i}(l, l) S-\sum_{j=0}^{r} B_{d j} R_{j} \prec 0, \\
& A_{\Theta s}(l, l) S-\sum_{j=0}^{r} B_{d j} R_{s j} \prec 0, \\
& L^{h} A_{\Theta i}(l, l+h) S-\sum_{j=0}^{r} L^{h} B_{d j} L^{h \mathrm{~T}} R_{j} \succ 0, \\
& L^{h} A_{\Theta s}(l, l+h) L^{h \mathrm{~T}} S-\sum_{j=0}^{r} L^{h} B_{d j} L^{h \mathrm{~T}} R_{s j} \succ 0,
\end{aligned}
$$

then the positive control gains $K, K_{s} \in \mathbb{R}_{+}^{r \times n}$ are given as

$$
\begin{gathered}
K_{d j}=R_{j} S^{-1}, \quad k_{j}^{\mathrm{T}}=l_{n}^{\mathrm{T}} K_{d j}, \quad K=\left[\begin{array}{c}
k_{1}^{\mathrm{T}} \\
\vdots \\
k_{r}^{\mathrm{T}}
\end{array}\right], \\
K_{s d j}=R_{s j} S^{-1}, \quad k_{s j}^{\mathrm{T}}=l_{n}^{\mathrm{T}} K_{s d j}, \quad K_{s}=\left[\begin{array}{c}
k_{s 1}^{\mathrm{T}} \\
\vdots \\
k_{s r}^{\mathrm{T}}
\end{array}\right]
\end{gathered}
$$

and force the strictly Metzler and Hurwitz matrices (39) and Metzler matrices $A_{c i}, A_{c s}$.

Hereafter, $*$ denotes the symmetric item in a symmetric matrix.

Proof. It quickly follows from (7), (14) that

$$
\left(A_{i}+A_{s}+\Delta_{i} A_{i}(t)+\Delta A_{s}(t)\right) S+S\left(A_{i}+A_{s}+\Delta A_{i}(t)+\Delta A_{s}(t)\right)^{\mathrm{T}} \prec 0,
$$

where $S \succ 0$ is a positive definite diagonal matrix. Combining associated parts of uncertainties in (50) it can write 


$$
\begin{aligned}
& \left(\Delta A_{i}(t)+\Delta A_{s}(t)\right) S+S\left(\Delta A_{i}(t)+\Delta A_{s}(t)\right) \\
= & M \Xi(t)\left(N_{a i}+N_{s}\right) S+S\left(N_{a i}+N_{s}\right)^{\mathrm{T}} \Xi(t)^{\mathrm{T}} M^{\mathrm{T}} \\
\preceq & \gamma^{-1} M \Xi(t) \Xi(t)^{\mathrm{T}} M^{\mathrm{T}}+\gamma S\left(N_{a i}+N_{s}\right)^{\mathrm{T}}\left(N_{a i}+N_{s}\right) S \\
\preceq & \gamma^{-1} M M^{\mathrm{T}}+\gamma S\left(N_{a i}+N_{s}\right)^{\mathrm{T}}\left(N_{a i}+N_{s}\right) S,
\end{aligned}
$$

where the final relation follows from (41) taking a positive scalar $\gamma \in \mathbb{R}_{+}$into account. Then, in the view of condition (50)

$$
\left(A_{i}+A_{s}\right) S+S\left(A_{i}+A_{s}\right)^{\mathrm{T}}+\gamma^{-1} M M^{\mathrm{T}}+\gamma S\left(N_{a i}+N_{s}\right)^{\mathrm{T}}\left(N_{a i}+N_{s}\right) S \prec 0
$$

and it is apparent that

$$
\left[\begin{array}{cc}
\left(A_{i}+A_{s}\right) S+S\left(A_{i}+A_{s}\right)^{\mathrm{T}}+\gamma^{-1} M M^{\mathrm{T}} & S\left(N_{a i}+N_{S}\right)^{\mathrm{T}} \\
\left(N_{a i}+N_{s}\right) S & -\gamma^{-1} I_{r}
\end{array}\right] \prec 0,
$$

when the Schur complement property is applied.

To govern the closed-loop system then (53) has to be satisfied with exchange for $A_{i} \leftarrow A_{c i}, A_{s} \leftarrow A_{c s}, N_{a i} \leftarrow N_{c a i}, N_{s} \leftarrow N_{c s}$ and $\delta=\gamma^{-1}$ that is

$$
\left[\begin{array}{cc}
\left(A_{c i}+A_{c s}\right) S+S\left(A_{c i}+A_{c s}\right)^{\mathrm{T}}+\delta M M^{\mathrm{T}} & S\left(N_{c a i}+N_{c s}\right)^{\mathrm{T}} \\
\left(N_{c a i}+N_{c s}\right) S & -\delta I_{r}
\end{array}\right] \prec 0 .
$$

Since it can check the following hold from (28)-(31)

$$
\begin{gathered}
A_{c i} S=A_{i} S-\sum_{j=1}^{r} b_{j} k_{j}^{\mathrm{T}} S=A_{i} S-\sum_{j=1}^{r} B_{d j} l_{n} l_{n}^{\mathrm{T}} K_{d j} S=A_{i} S-\sum_{j=1}^{r} B_{d j} l_{n} l_{n}^{\mathrm{T}} R_{j}, \\
A_{c s} S=A_{S} S-\sum_{j=1}^{r} b_{j} k_{s j}^{\mathrm{T}} S=A_{s} S-\sum_{k=1}^{m} B_{d j} l_{n} l_{n}^{\mathrm{T}} K_{s d j} S=A_{s} S-\sum_{j=1}^{r} B_{d j} l_{n} l_{n}^{\mathrm{T}} R_{s j}, \\
N_{c a i} S=N_{a i} S-\sum_{j=1}^{r} n_{b j} k_{j}^{\mathrm{T}} S=N_{a i} S-\sum_{j=1}^{r} N_{b d j} l_{r} l_{n}^{\mathrm{T}} K_{d j} S=N_{a i} S-\sum_{j=1}^{r} N_{b d j} l_{r} l_{n}^{\mathrm{T}} R_{j}, \\
N_{c S} S=N_{s} S-\sum_{j=1}^{r} n_{b j} k_{s j}^{\mathrm{T}} S=N_{s} S-\sum_{j=1}^{r} N_{b d j} l_{r} l_{n}^{\mathrm{T}} K_{s d j} S=N_{s} S-\sum_{j=1}^{r} N_{b d j} l_{r} l_{n}^{\mathrm{T}} R_{s j} S,
\end{gathered}
$$

where

$$
R_{j}=K_{d j} S, \quad R_{s j}=K_{s d j} S .
$$

then LMI (57) conditioned by (58)-(59) imply (43).

The inequalities (17), (18) and the relation (19) in turn give the similar expressions for actual parametric constraints

$$
\begin{gathered}
A_{\Theta i}(l, l)-\sum_{j=0}^{r} B_{d j} K_{d j} \prec 0, \quad A_{\Theta s}(l, l)-\sum_{j=0}^{r} B_{d j} K_{s d j} \prec 0, \\
A_{\Theta i}(l, l+h)-\sum_{j=0}^{r} B_{d j} K_{d j h} \succ 0, \quad A_{\Theta s}(l, l+h)-\sum_{j=0}^{r} B_{d j} K_{s d j h} \succ 0,
\end{gathered}
$$

where

$$
K_{d j h}=L^{h \mathrm{~T}} K_{d j} L^{h}, \quad K_{s d j h}=L^{h \mathrm{~T}} K_{s d j} L^{h} .
$$

Then, multiplying the right side of (60) by $S$ it yields

$$
A_{\Theta i}(l, l) S-\sum_{j=0}^{r} B_{d j} K_{d j} S \prec 0, \quad A_{\Theta s}(l, l) S-\sum_{j=0}^{r} B_{d j} K_{s d j} S \prec 0
$$

and replacing with (59) then (60) imply (44) and (45). 
Pre-multiplying the left side by $L^{h}$ and post-multiplying the right side by $L^{h \mathrm{~T}} S$ then (61) is rewritten as

$$
A_{\Theta i}(l, l+h) S-\sum_{j=0}^{r} B_{d j} K_{d j h} L^{h \mathrm{~T}} S \succ 0, \quad A_{\Theta s}(l, l+h) S-\sum_{j=0}^{r} B_{d j} K_{s d j h} L^{h \mathrm{~T}} S \succ 0,
$$

Thus, substituting (62)

$$
A_{\Theta i}(l, l+h) S-\sum_{j=0}^{r} B_{d j} L^{h \mathrm{~T}} K_{d j} S \succ 0, \quad A_{\Theta s}(l, l+h) S-\sum_{j=0}^{r} B_{d j} L^{h \mathrm{~T}} K_{s d j} S \succ 0,
$$

where $L^{h} L^{h \mathrm{~T}}=I_{n}$ and replacing with (59) then (64) imply (46) and (47). This completes the proof.

Remark 4. If the set of LMIs (42)-(47) is feasible, the conditions (44)-(47) cover that the matrices $A_{c i}, A_{i}$ are strictly Metzler, the matrix inequality (43) clamps that $A_{c i}^{\circ}$ is Metzler and Hurwitz and the positive definite diagonal matrix variables $S, R_{j}, R_{s j}$ guaranty that $K, K_{s} \in \mathbb{R}_{+}^{r \times n}$ are positive. Thus, the given problem can be solved by (42)-(47), guaranteing quadratically stable closed-loop system.

Potentially, the given task formulation can be extended for models with the interval uncertainties in time-delays and inputs using the concept of parallel distributed compensation [25].

Corollary 1. Consider that $B \in \mathbb{R}_{+}^{n \times r}$ is nonnegative and that $A_{i}, A_{s} \in \mathbb{M}_{-+}^{n \times n}$ are pure Metzler but all are mutually compatible in the position of a zero value off-diagonal element (zero element falls in all these matrices on the position with the row number $z$ and the column number $y$ ).

If the $z$-th row of $B$ is zero vector $\left(b_{z}^{T}=0^{\mathrm{T}}\right)$, the design conditions (42)-(47) remain valid and the results in the feasible case are positive $K, K_{s}$, pure Metzler matrices $A_{c i}, A_{s} \in \mathbb{M}_{-+}^{n \times n}$ and pure Metzler and Hurwitz $A_{c i}^{\circ} \in \mathbb{M}_{-+}^{n \times n}$.

If elements of the $z$-th rov of $B$ are nonzero and an off-diagonal $(z, y)$-element is equal to zero in mutually compatible $A_{i}, A_{s} \in \mathrm{M}_{-+}^{n \times n}$ then the design conditions have to be reformulated for the structural positive semi-definite diagonal matrix variables $R_{j}, R_{s j}$, defined as

$$
\begin{gathered}
R_{j}=\operatorname{diag}\left[\begin{array}{lllllll}
r_{j 1} & \cdots & r_{j, z-1} & r_{j z} & r_{j, z+1} & \cdots & r_{j n}
\end{array}\right] \succeq 0 \\
R_{s j}=\operatorname{diag}\left[\begin{array}{lllllll}
r_{s, j 1} & \cdots & r_{s, j, z-1} & r_{s j z} & r_{s, j, z+1} & \cdots & r_{s, j n}
\end{array}\right] \succeq 0
\end{gathered}
$$

where for all $j$ and given $z$

$$
r_{j z}=0, \quad r_{s j z}=0
$$

The results in the last noted case guaranty nonnegative $K, K_{s}$, pure Metzler and Hurwitz $A_{c i}^{\circ} \in \mathbb{M}_{-+}^{n \times n}$ and pure Metzler matrices $A_{i}, A_{s} \in \mathbb{M}_{-+}^{n \times n}$. The inclusion of the reference [35] makes up for the omitted details of the explanation.

Other cases, if they are solvable, can be done "ad hoc" in definition of distinct structural positive semi-definite diagonal matrix variables $R_{j}, R_{s j}$ for different $j \in\langle 1, s\rangle$.

The aim of the proposed methodology is to stabilize the systems of the given class at the defined parameters of the system model. In the solved task, the task validation is included inherently in the synthesis conditions - if the considered system is stabilizable, there must be positive definite diagonal matrix variables such that the proposed set of LMI is feasible. Only if the system has uncontrollable modes the validation would be 
more involved. For the control approach, the quadratic stability of the overall closed-loop error dynamics is proven using by the system matrix eigenvalue emplacement criterion.

Remark 5. For application use in the control theory, this type of design task does not define the principle of LMI algorithm implementation, but only their boundaries [36]. The final characteristics are defined by the choice of a standard LMI solver, which defines the computational complexity of the implemented algorithms. In this case the algorithm solves the design task in polynomial time with respect to the cardinality of the state space, the number of matrix variables and applied LMI solver. Using the LMI solver SeDuMi the computational complexity is $O\left(h_{d v}^{2} h_{a r}^{2.5}+h_{a r}^{3.5}\right)$, where $h_{d v}$ is the number of decision variables and $h_{\text {ar }}$ is the number of active rows in the LMIs [37], while the computational complexity of MATLAB LMI Toolbox is $O\left(h_{d v}^{3} h_{a r}\right)$ [38]. The former algorithm is so more efficient for problems with a large number of LMI variables.

Because the principle is not intended for use in an adaptive control structure with the real-time control parameter calculation, this computational complexity is not applicable limiting.

The proposed method provides a novel, original, constructive and nontrivial formulation, where the computation of the control gains reduces to a feasibility problem over a set of LMIs. The solution depends on the positive parameter $\delta$, corresponding to an upper bound for the matching uncertainty condition and is optimized in the nominally defined design task. The parameter $\delta$ can be fixed in the design step interactively in the case it is necessary to modify (usually slow down) the dynamics of a closed loop.

\section{Illustrative Example}

To illustrate the effectiveness of the proposed method the design principle is applied to the system model (1), (2) defined by the system parameters

$$
\begin{aligned}
& A_{1}=\left[\begin{array}{rrr}
-0.27 & 1.94 & 1.45 \\
0.08 & -3.96 & 0.20 \\
0.45 & 0.70 & -2.91
\end{array}\right], A_{2}=\left[\begin{array}{rrr}
-0.26 & 2.06 & 1.55 \\
0.14 & -3.64 & 0.36 \\
0.25 & 0.78 & -2.55
\end{array}\right], L^{\mathrm{T}}=\left[\begin{array}{ll}
0 & I_{2} \\
1 & 0^{\mathrm{T}}
\end{array}\right], l_{r}=\left[\begin{array}{l}
1 \\
1
\end{array}\right], \\
& A_{s}=\left[\begin{array}{rrr}
-0.01 & 0.12 & 0.15 \\
0.01 & -0.38 & 0.03 \\
0.04 & 0.07 & -0.31
\end{array}\right], B=\left[\begin{array}{ll}
0.5 & 1.0 \\
1.0 & 0.9 \\
0.7 & 1.1
\end{array}\right], C^{\mathrm{T}}=\left[\begin{array}{ll}
1 & 0 \\
0 & 1 \\
0 & 0
\end{array}\right], l_{n}=\left[\begin{array}{l}
1 \\
1 \\
1
\end{array}\right], M=\left[\begin{array}{ll}
0 & 0 \\
1 & 0 \\
0 & 1
\end{array}\right] \text {, } \\
& N_{a 1}=\left[\begin{array}{ccc}
0.02 & 0 & 0 \\
0 & 0.01 & 0
\end{array}\right], N_{a 2}=\left[\begin{array}{ccc}
0.01 & 0 & 0 \\
0 & 0.02 & 0
\end{array}\right], N_{s}=\left[\begin{array}{ccc}
0.02 & 0 & 0 \\
0 & 0.02 & 0
\end{array}\right], N_{b}=\left[\begin{array}{cc}
0.2 & 0 \\
0 & 0.2
\end{array}\right] \text {, }
\end{aligned}
$$

where $A_{1}, A_{2}, A_{s}$ are strictly Metzler but not Hurwitz.

With the synthesis focusing points, of immediate consequences are primary the common matrix auxiliary parameters

$$
\begin{aligned}
& A_{\Theta 1}(l, l)=-\operatorname{diag} 1\left[\begin{array}{lll}
0.27 & 3.96 & 2.91
\end{array}\right], \quad A_{\Theta 2}(l, l)=-\operatorname{diag}\left[\begin{array}{llll}
0.26 & 3.64 & 2.55
\end{array}\right], \\
& A_{\Theta 1}(l, l+1)=\operatorname{diag}\left[\begin{array}{lll}
1.94 & 0.20 & 0.45
\end{array}\right], \quad A_{\Theta 2}(l, l+1)=\operatorname{diag}\left[\begin{array}{lll}
2.06 & 0.36 & 0.25
\end{array}\right] \text {, } \\
& A_{\Theta 1}(l, l+2)=\operatorname{diag}\left[\begin{array}{lll}
1.45 & 0.08 & 0.70
\end{array}\right], \quad A_{\Theta 2}(l, l+2)=\operatorname{diag}\left[\begin{array}{lll}
1.55 & 0.14 & 0.78
\end{array}\right] \text {, } \\
& A_{\Theta s}(l, l)=-\operatorname{diag}\left[\begin{array}{lll}
0.01 & 0.38 & 0.31
\end{array}\right] \text {, } \\
& A_{\Theta s}(l, l+1)=\operatorname{diag}\left[\begin{array}{lll}
0.12 & 0.03 & 0.04
\end{array}\right], \quad A_{\Theta s}(l, l+2)=\operatorname{diag}\left[\begin{array}{lll}
0.15 & 0.01 & 0.07
\end{array}\right], \\
& B_{d 1}=\operatorname{diag}\left[\begin{array}{lll}
0.5 & 1.0 & 0.7
\end{array}\right], \quad B_{d 2}=\operatorname{diag}\left[\begin{array}{lll}
1.0 & 0.9 & 1.1
\end{array}\right] \text {, }
\end{aligned}
$$




$$
N_{b d 1}=\operatorname{diag}\left[\begin{array}{ll}
0.2 & 0
\end{array}\right], \quad N_{b d 2}=\operatorname{diag}\left[\begin{array}{ll}
0 & 0.02
\end{array}\right] .
$$

Solving (42)-(47), applying SeDuMi package in MATLAB environment, the feasible task admits the solution with the following positive definite diagonal matrix variables

$$
S=\operatorname{diag}\left[\begin{array}{lll}
3.3558 & 0.4667 & 0.6948
\end{array}\right],
$$

$$
\begin{aligned}
& R_{1}=\operatorname{diag}\left[\begin{array}{lll}
0.0879 & 0.1008 & 0.0422
\end{array}\right], \quad R_{2}=\operatorname{diag}\left[\begin{array}{llll}
0.1190 & 0.0813 & 0.0496
\end{array}\right], \\
& R_{s 1}=\operatorname{diag}\left[\begin{array}{lll}
0.0115 & 0.0132 & 0.0066
\end{array}\right], \quad R_{s 2}=\operatorname{diag}\left[\begin{array}{lll}
0.0119 & 0.0093 & 0.0070
\end{array}\right] \text {. }
\end{aligned}
$$

Using relations (48), (49) the positive gain matrices of the common control law are computed as

$$
K=\left[\begin{array}{lll}
0.0023 & 0.0426 & 0.0078 \\
0.0079 & 0.0208 & 0.0167
\end{array}\right], \quad K_{s}=\left[\begin{array}{lll}
0.0296 & 0.1861 & 0.0614 \\
0.0352 & 0.1583 & 0.0659
\end{array}\right]
$$

and it is trivial to construct the matrices $A_{c 1}^{\circ}, A_{c 2}^{\circ}$

$$
\begin{aligned}
& A_{c 1}^{\circ}=\left[\begin{array}{rrr}
-0.3338 & 1.7437 & 1.4834 \\
0.0253 & -4.7589 & 0.0865 \\
0.4264 & 0.3854 & -3.3588
\end{array}\right], \quad \rho\left(A_{c 1}^{\diamond}\right)=\left[\begin{array}{l}
-0.1231 \\
-3.5463 \\
-4.7821
\end{array}\right], \\
& A_{c 2}^{\circ}=\left[\begin{array}{rrr}
-0.3238 & 1.8637 & 1.5834 \\
0.0853 & -4.4389 & 0.2465 \\
0.2264 & 0.4654 & -2.9988
\end{array}\right], \quad \rho\left(A_{c 2}^{\diamond}\right)=\left[\begin{array}{l}
-0.1459 \\
-3.0848 \\
-4.5308
\end{array}\right] .
\end{aligned}
$$

The design condition guaranties only that $A_{c 1}, A_{c 2}, A_{c s}$ be strictly Metzler, but for such given system parameters it is obtained

$$
\begin{aligned}
& A_{c 1}=\left[\begin{array}{rrr}
-0.3186 & 1.6578 & 1.3483 \\
0.0219 & -4.3328 & 0.0750 \\
0.3927 & 0.3572 & -3.0310
\end{array}\right], \quad \rho\left(A_{c 1}\right)=\left[\begin{array}{l}
-0.1226 \\
-3.2068 \\
-4.3529
\end{array}\right], \\
& A_{c 2}=\left[\begin{array}{rrr}
-0.3086 & 1.7778 & 1.4483 \\
0.0819 & -4.0128 & 0.2350 \\
0.1927 & 0.4372 & -2.6710
\end{array}\right], \quad \rho\left(A_{c 2}\right)=\left[\begin{array}{l}
-0.1452 \\
-2.7440 \\
-4.1032
\end{array}\right], \\
& A_{c s}=\left[\begin{array}{rrr}
-0.0153 & 0.0859 & 0.1352 \\
0.0034 & -0.4262 & 0.0115 \\
0.0337 & 0.0283 & -0.3277
\end{array}\right], \quad \rho\left(A_{c s}\right)=\left[\begin{array}{l}
-0.0003 \\
-0.3396 \\
-0.4293
\end{array}\right],
\end{aligned}
$$

which all are strictly Metzler and Hurwitz, but this results is not guaranteed in general.

One can verify that using slightly different parameter matrix with the time-delay relation $A_{s}$ in the form

$$
A_{s}=\left[\begin{array}{rrr}
-0.01 & 0.12 & 0.15 \\
0.01 & -0.38 & 0.03 \\
0.04 & 0.07 & -0.31
\end{array}\right] \longleftarrow\left[\begin{array}{rrr}
-0.01 & 0.12 & 0.15 \\
0.01 & -0.38 & 0.03 \\
0.04 & 0.07 & -0.30
\end{array}\right],
$$

the solution mean strictly Metzler and Hurwitz $A_{c 1}^{\circ}, A_{c 2}^{\circ}, A_{c 1}, A_{c 2}$, but $A_{c s}$ is only strictly Metzler, since in this case

$$
A_{c s}=\left[\begin{array}{rrr}
-0.0153 & 0.0859 & 0.1352 \\
0.0034 & -0.4262 & 0.0115 \\
0.0337 & 0.0283 & -0.3177
\end{array}\right], \quad \rho\left(A_{c s}\right)=\left[\begin{array}{r}
0.0001 \\
-0.3302 \\
-0.4291
\end{array}\right] .
$$

Note, the matrix $A_{c s}$ does not need to be a Hurwitz matrix since the main idea is to construct a stable augmented matrix $A_{c i}^{\circ}$.

Real negative system eigenvalues are conditional on the use of positive Metzler systems because they guarantee the aperiodic positive trajectories of state variables for a 
non-negative initial state of the system with convergence to the system equilibrium only in the positive subspace $\mathbb{R}_{+}^{n}$. For this reason, the graphical presentation of state trajectories appears to be redundant. The given example contains all the parameters so that interesting readers can verify this fact.

Note, the potentially comparable method for stabilization of time-delay positive continuous-time systems presented in [39] produces in general the non-negative gains and the nonnegative closed-loop system matrices although the description of the system is based on the strictly Metzler matrix structures. As a result, there is no comparison base of the design methods for the results presented in this paper for uncertain Mezler-TakagiSugeno time-delay systems.

For sake of readability, in the example is only treated the case of strictly Metzler system matrices, but the authors are convinced that this explicit example helps interesting readers in understanding such defined design problem in the whole complexity.

\section{Concluding Remarks}

In the paper, a novel method is proposed for design of control law parameters for uncertain Mezler-Takagi-Sugeno time-delay systems with a constant point time-delay in state and it is showed that it preserves principle of diagonal stabilization. Structural properties of the considered systems with uncertainties are reflected to ensure the closed-loop quadratic stability when defining the LMIs structure which cover the system structural constraints, the parameter uncertainties and the point-delay in system state. What seems perhaps specific is additional conditions to find state feedbacks with positive system gains, preserving the closed-loop system positivity for strictly Metzler system matrices. The parameter uncertainty domination as well as quadratic stability are guaranteed by using the proposed structure of the state feedback. The illustrative example confines to the theoretical starting points. To the best of authors' knowledge, no comparable theoretical results are available for design of state control of this class of systems with strictly Metzler matrix structures.

The reason justifying the use of uncertain Metzler-Takagi-Sugeno time-delay model is the need to minimize potentially the time-delay impact on the control with distributed sensor structures of nonlinear systems. In this sense the account of the uncertainties in Metzler-Takagi-Sugeno models allows adjusting the system state so that the system parameter constraints imposed by the system positivity are satisfied. This is also the main topic for the future research.

The limitation of the presented structure of LMIs is tied to the positive definiteness of the diagonal variables $R_{j}, R_{s j}$, which in the case of the purely Metzler structure of the matrices may mean a marginal feasibility of the solution. Corollary 1 outlines an approach of solving this problem by defining these variables as structured in an ad hoc manner for a specific case. Another disadvantage limiting their widespread use is the problem of selecting the nonnegative uncertainty matching conditions.

Solving under given system parametric constraints and the boundlessness of system uncertainties substantially limits a control parameter tuning by reexpressing the design conditions with an additive tuning parameter. However, such a path must be used for uncertain Metzler-Takagi-Sugeno time-delay systems with a polytopic region of uncertainty as this introduces another scalar tuning parameter when using the slack matrix 
principle. If an unknown disturbance is acting on the uncertain Metzler-Takagi-Sugeno time-delay systems, adjusting to the $\mathrm{H}_{\infty}$ norm of the disturbance transfer function must be done by even more tuning scalar parameter. The ideas presented can be potentially extended to a more general class of Mezler-Takagi-Sugeno time-delay systems with interval time-delays or to design a non-fragile memory feedback controller for the considered system class and to the system reference trajectory problem. These cases are partly a topic for future research.

\section{Acknowledgements}

The work presented in the paper was supported by VEGA, the Grant Agency of the Ministry of Education and the Academy of Science of Slovak Republic, under Grant No. $1 / 0463 / 21$. This support is very gratefully acknowledged.

\section{References}

[1] Zheng F, Frank P.M. Robust control of uncertain distributed delay systems with application to the stabilization of combustion in rocket motor chambers. Automatica. 2002; 38(3):487-497.

[2] Hale JK, Lunel S.M.V. Introduction to Functional Differential Equations. New York: Springer; 1993, $458 \mathrm{p}$.

[3] Wang Y, Zou X. On a predator-prey system with digestion delay and anti-predation strategy. Journal of Nonlinear Science. 2020, 30:1579-605.

[4] Fridman E. Introduction to Time-Delay Systems: Analysis and Control, Cham: Springer Nature; 2014 $378 \mathrm{p}$.

[5] Li X, Yurkovich S. Sliding mode control of delayed systems with application to engine idle speed control. IEEE Transactions on Control Systems Technology. 2001, 9(6):802-10.

[6] Miller DA, Rossi, M. Simultaneous stabilization with near pptimal LQR performance. In: Proceedings of the 38th Conference on Decision \& Control; 1999 December 07-10; Woenix, Arizona, USA: p. 23005 .

[7] Geromel JC, Peres PLD, Souza SR. $\mathrm{H}_{2}$ guaranteed cost control for uncertain continuous-time linear systems. Systems \& Control Letters. 1992; 19(1):23-27.

[8] Khargonekar PP, Petersen IR, Zhou K. Robust stabilization of uncertain systems and $\mathrm{H}_{\infty}$ optimal control. IEEE Transactions on Automatic Control. 1990; 35(3): 356-61.

[9] Xu S, Lam J, Yang C. Quadratic stability and stabilization of uncertain linear discrete-time systems with state delay. Systems \& Control Letters, 2001; 43(1):77-84.

[10] Cao SG, Rees NW, Feng G. $\mathrm{H}_{\infty}$ control of uncertain fuzzy continuous-time systems. Fuzzy Sets and Systems. 2020; 115:171-90.

[11] Nikaido H. Convex Structures and Economic Theory. New York: Academic Press; 1968, 422 p.

[12] Smith H.L. Monotone Dynamical Systems: An Introduction to the Theory of Competitive and Cooperative Systems. Providence: American Mathematical Society; 1995, 178 p.

[13] Hmamed A, Ait Rami M, Benzaouia A, F. Tadeo F. Stabilization under constrained states and controls of positive systems with time delays. European Journal of Control. 2012; 18(2):182-90.

[14] Anderson BDO. Positive system realizations. In: Open Problems in Mathematical Systems and Control Theory. London: Springer; 1999, p. 7-10.

[15] Haddad W.M, Chellaboina V. Stability theory for nonnegative and compartmental dynamical systems with time delay. Systems \& Control Letters. 2004; 51(5):355-61.

[16] Liu X, Lam J. Relationships between asymptotic stability and exponential stability of positive delay systems. International Journal of General Systems. 2013; 42(2):224-38.

[17] Farina L, Rinaldi S. Positive Linear Systems: Theory and Applications. New York: John Wiley \& Sons; 2000, 300 p.

[18] Berman A, Neumann M, Stern R. Nonnegative Matrices in Dynamic Systems. New York: John Wiley \& Sons, New York; 1989, 167 p. 
[19] Peet MM, Papachristodoulou A, Lall S. Positive forms and stability of linear time-delay system. SIAM Journal on Control and Optimization. 2009; 47(6):3237-58.

[20] Takagi T, Sugeno M. Fuzzy identification of systems and its applications to modeling and control. IEEE Transactions on Systems, Man, and Cybernetics. 1985; 15(1):116-32.

[21] Liu X. Constrained control of positive systems with delays. IEEE Transactions on Automatic Control. 2009; 54(7):1596-600.

[22] Krokavec D, Filasová A. On observer design methods for a class of Takagi-Sugeno fuzzy systems. Computer Science \& Information Technology. 2014; 4(11):279-90.

[23] Vanderbei RJ. Linear Programming: Foundations and Extensions. Cham: Springer Nature; 2020, 466 p.

[24] Tanaka K, Wang, HO. Fuzzy Control Systems Design and Analysis: A Linear Matrix Inequality Approach. New York: John Wiley \& Sons; 2001. 305 p.

[25] Krokavec D, Filasová A. Optimal fuzzy control for a class of nonlinear systems. Mathematical Problems in Engineering. 2012; 2012:1-29.

[26] Ait Rami M, Helmke U, Tadeo F. Positive observation problem for linear time-delay positive systems. In: Proceedings of the 15th Mediterranean Conference on Control \& Automation MED2007; 2007 June 27-29; Athens, Greece: p. 1-6.

[27] Gallier J. Geometric Methods and Applications: For Computer Science and Engineering. New York: Springer; 2011, 709 p.

[28] Ebihara Y. Stability analysis of neutral type time-delay positive systems with commensurate delays. IFAC PapersOnLine. 2017; 50(1):3093-98.

[29] Briat, C. Stability and performance analysis of linear positive systems with delays using input-output methods. International Journal of Control. 2018; 91(7):1669-92.

[30] Mason O. Diagonal Riccati stability and positive time-delay systems. Systems \& Control Letters. 2012; 61(1):6-10.

[31] Horn RA, Johnson C.R. Matrix Analysis. New York: Cambridge University Press; 1985, 643 p.

[32] Krokavec D, Filasová A. LMI based principles in strictly Metzlerian systems control design. Mathematical Problems in Engineering. 2018; 2018:1-14.

[33] Krokavec D, Filasová A. Control design for linear strictly Metzlerian descriptor systems. In: Proceedings of the 18th EUCA European Control Conference ECC2020; 2020 May 12-15; Saint-Petersburg, Russia: p. 2092-97.

[34] Amato A, Pironti A, Scala S. Necessary and sufficient conditions for quadratic stability and stabilizability of uncertain linear time-varying systems. IEEE Transactions on Automatic Control. 1996; 41(1):125-28.

[35] Krokavec D, Filasová A. $\mathrm{H}_{\infty}$ norm principle in residual filter design for discrete-time linear positive systems. European Journal of Control. 2019; 45:17-29.

[36] VanAntwerp JG, Braatz RD. A tutorial on linear and bilinear matrix inequalities. Journal of Process Control. 2000; 10(4):363-85.

[37] Peaucelle D, Henrion D, Labit Y, Taitz K. User's Guide for SeDuMi Interface. Toulouse: LAAS-CNRS; 2002, 37 p.

[38] Gahinet PM, Nemirovski A, Laub AJ, Chilali M. LMI Control Toolbox User's Guide. Natick: The MathWorks; 1995, 357 p.

[39] Elloumi W, Benzaouia A, Chaabane M. Delay-dependent stabilization conditions of controlled positive continuous-time systems. International Journal of Automation and Computing. 2014; 11(6):653-60. 